\title{
EDUCACIÓN SUPERIOR NO UNIVERSITARIA PERUANA: LA GESTIÓN DE TRÁMITES Y SU IMPLICANCIA EN LA INSERCIÓN LABORAL
}

\author{
PERUVIAN NON-UNIVERSITY HIGHER EDUCATION: PAPERWORK \\ MANAGEMENT AND ITS IMPLICATION IN JOB PLACEMENTT \\ (D) Vilma María Fe Bellido De La Cruz ${ }^{1 *}$ \\ bellido.unsch@gmail.com \\ ${ }^{1}$ Universidad Nacional San Cristóbal de Huamanga; Ayacucho, Perú
}

*Correspondencia: Vilma María Fe Bellido De La Cruz. Email: bellido.unsch@gmail.com

Recibido: 19.08.2020 | Aprobado: 10.10.2020

\section{RESUMEN}

El objetivo fue analizar la gestión de trámites y su implicancia en la inserción laboral de los egresados del Instituto de Educación Superior Tecnológico Privado Avansys; para lograrlo se utilizó la metodología de la evaluación cualitativa con El Cambio Más Significativo. Evidenciando que el $27.3 \%$ fueron mejor calificados para un puesto laboral al contar con el título y el $84.6 \%$ lo considera necesario obtener el título para insertarse laboralmente. Se concluyó que la gestión de título del instituto obstaculiza la inserción laboral a los egresados. El factor que dificulta el proceso es el elevado costo y la demora del trámite que excede el tiempo establecido.

Palabras clave: Gestión de trámites, inserción laboral, educación no universitaria.

\section{ABSTRACT}

The objective was to analyze the management of procedures and its implication in the labor insertion of graduates of the Avansys Private Technological Higher Education Institute; To achieve this, the methodology of qualitative evaluation with The Most Significant Change was used. Evidence that $27.3 \%$ were better qualified for a job position by having the title and $84.6 \%$ consider it necessary to obtain the title to enter the job market. It was concluded that the management of the institute's degree hinders the employment of graduates. The factor that makes the process difficult is the high cost and the delay in the process that exceeds the established time.

Keywords: Management of procedures, labor insertion, nonuniversity education. 


\section{INTRODUCCIÓN}

Una de las principales actividades del ser humano, que contribuye a su autorrealización y al desarrollo de la sociedad, es el trabajo e insertarse al mercado laboral. Para ello, el nivel de estudios alcanzado es una ventaja competitiva y resulta determinante para disminuir el desempleo (García, 2010).

La educación superior, según la Ley 28044 (28 de julio de 2003), es:

"La segunda etapa del Sistema Educativo que consolida la formación integral de las personas, produce conocimiento, desarrolla la investigación e innovación y forma profesionales en el más alto nivel de especialización y perfeccionamiento en todos los campos del saber, el arte, la cultura, la ciencia y la tecnología a fin de cubrir la demanda de la sociedad y contribuir al desarrollo y sostenibilidad del país". (artículo 49)

De acuerdo a la Ley citada, las instituciones de educación superior incluyen a las instituciones universitarias, así como los institutos, escuelas y otros centros públicos o privados. Para ingresar a la educación superior es obligatorio haber finalizado la educación básica.

En el Perú, según INEI (2017), el 13.1 \% de la población en edad en trabajar cuenta con un nivel superior no universitario y un $16.4 \%$ con universitario. Asimismo, la tasa de desempleo, solo en Lima Metropolitana, en este mismo año, fue de $6.7 \%$. El desempleo de la población en edad de trabajar, con nivel superior no universitaria, está relacionado con diversos factores, entre ellos el bajo desempeño académico de las instituciones, la demanda del mercado laboral y la demora en obtención del título por diversas razones.

Los desafíos que se plantean a la educación superior son diversos. Uno de ellos es reducir las barreras burocráticas para que los jóvenes se inserten oportunamente al mercado laboral. A este escenario se suma la desigualdad en oportunidades y logros educacionales; bajos estándares de enseñanza y niveles de aprendizaje; limitadas habilidades sociales; escasa preparación de práctica; dificultades de atención y adaptación a horarios por carga laboral (Chacaltana y Sulmot, 2004). Si estos factores no son atendidos en su debido momento, reducirán las posibilidades en la inserción laboral y en la carrera que han sido formados.

La inserción laboral es el proceso de incorporación de las personas a la actividad económica, en su mayoría jóvenes, independiente al nivel educativo logrado o la especialización conseguida (Produce, 2015)

El nivel de inserción en el campo laboral entre los egresados es mayor en comparación a las egresadas; el 75\% de las egresadas no desempeñan su carrera; lo que indica que los egresados tienen mayor oportunidad para trabajar en instituciones tanto privadas, como estatales; desempeñándose en distintos cargos, independientemente de lo estudiado; en cambio, la mayoría de las egresadas no desempeñan su carrera, debido a muchos factores, siendo uno de ellos el factor familiar por lo que no trabajan; ya que algunas se casan, o tienen hijos, lo que les impide laborar (Arévalo, 2016)

En relación a la situación laboral de egresados de IEST ¿Qué hubieras hecho al culminar secundaria, sin apoyo económico? 'Pues, un año estudiar, un año trabajar" 
Indica José Miguel (29), egresado del instituto Avansys - quien estudió mecánica automotriz y ahora trabaja como chofer." muchos soñamos trabajar en la carrera que hemos estudiado, pero eso no ocurre, la realidad es otra, tienes que adecuarte al trabajo que se te presenta y no el trabajo a ti”

Lo que él ignora quizá es que 7 de cada 10 jóvenes en Perú (70\%) hace exactamente eso, trabajar tras salir de la secundaria sin recibir una educación superior. Así lo revela una investigación del Consejo Privado de Competitividad (2018)

\section{METODOLOGÍA}

Se utilizó la metodología de evaluación cualitativa mediante el Cambio Más Significativo - CMS (Davies y Dart, 2005). Los pasos de esta metodología están inmersos en la planificación, recolección de datos, sistematización y comunicación de resultados.

Se realizó la recolección de información mediante historias de vida a partir de 4 preguntas: (i) ¿cómo te ayudó tu título para insertarte laboralmente?; (ii) ¿fue necesario obtener tu título para que puedas insertarte laboralmente?; (iii) ¿alguna vez se te fue negada una oportunidad laboral por no tener tu título?; y (iv) ¿cuáles fueron las dificultades que encontraste para obtener tu título?

La unidad de análisis se enmarca en informantes clave de los involucrados en institutos de educación superior. Esto se debe a que el universo de estudio son los involucrados de la misma institución. Se consideró como tamaño de muestra 20 egresados. Si bien la selección de muestra fue aleatoria, se procuró que el grupo sea mixto respecto al sexo, puesto que, aunque no fue el objetivo, contribuye en razón a la igualdad de género.

En relación con las técnicas de recojo de información se desarrolló una ficha de historias de vida como testimonios directos de la situación de los actores clave, a partir de los que se generaron datos estadísticos cualitativos.

\section{RESULTADOS Y DISCUSIÓN}

Las tareas principales de acuerdo con la etapa de desarrollo de las personas en edad de trabajar son insertarse al mercado laboral, considerando que el nivel de estudios alcanzado por las personas sí es determinante para disminuir el desempleo (García, 2010). Para tal fin es necesario realizar estudios en educación superior universitaria o no universitaria y conseguir el título que permita el acceso a un puesto laboral.

Respecto a la importancia del título para la inserción laboral, en la Figura 1 se observa que el $84.6 \%$ lo considera necesario y el $15.4 \%$ señala lo contrario. 


\section{Figura 1}

Titulo en la inserción

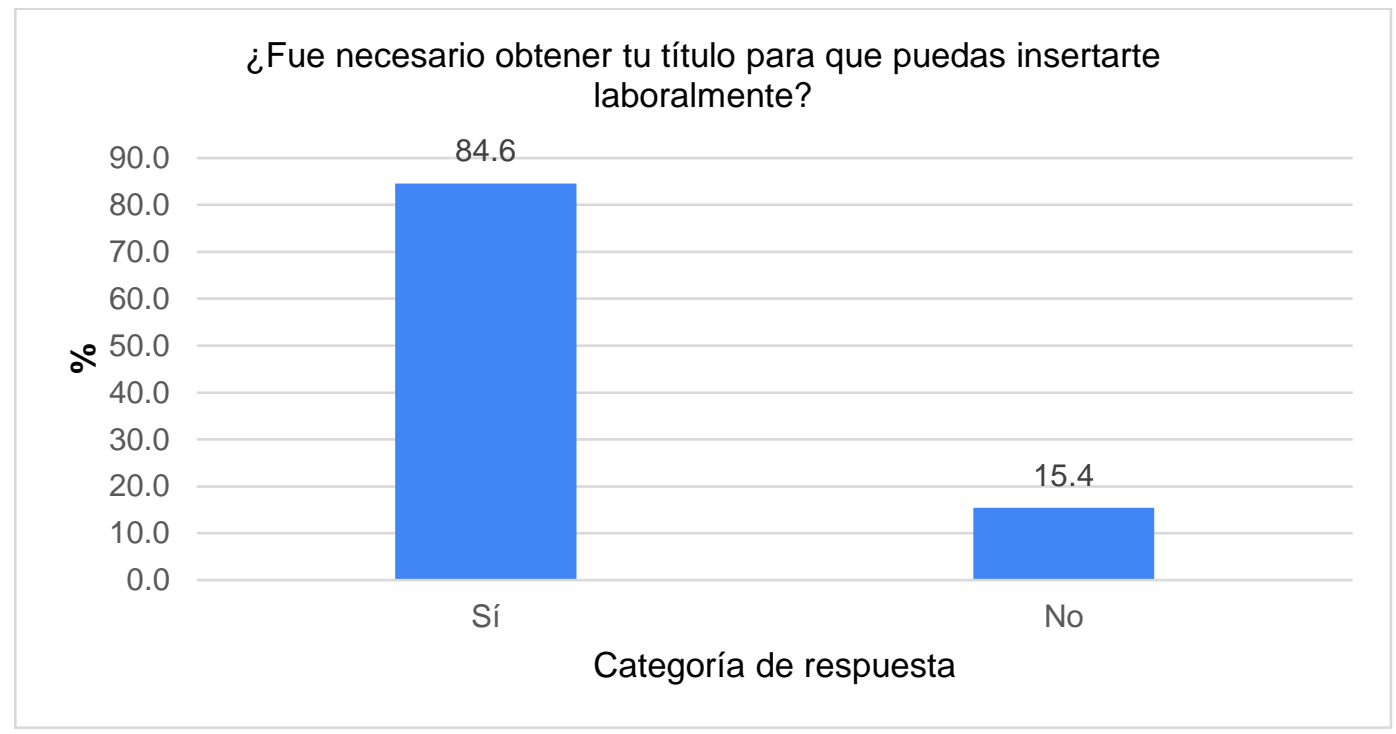

En la Ley $\mathrm{N}^{\circ} 28044$ se incide en que la educación superior consolida la formación integral de las personas, produce conocimiento, desarrolla la investigación para cubrir la demanda de la sociedad, y contribuir al desarrollo y sostenibilidad del país; asimismo en la Ley $\mathrm{N}^{\circ} 29394$ resalta la formación de manera integral a los profesionales en el arte, la cultura, la ciencia y la tecnología y sintetiza en el Decreto Supremo No 004-2010-ED que los Institutos y Escuelas de Educación Superior Tecnológicos, públicas o privadas ofertarán carreras tomando en cuenta los requerimientos del sector productivo y del desarrollo local, regional y nacional, y sus planes deben vincular con el sector productivo y la inserción laboral de sus egresados.

María, egresada de Avansys, comenta: "Cuando egresé del instituto de la carrera de contabilidad, al no tener el titulo acepté los trabajos que se presentaron y como no tenía tiempo para tramitar y cumplir los requisitos lo dejé, ahora bago logística en una empresa, me bubiera gustado trabajar en lo que me formé, tal vez. cuando saque mi titulo"

La lógica de una Institución Educativa Superior Tecnológico (IEST) es que sus profesionales técnicos especialistas deberían trabajar para lo que se formaron y ser capaces de responder a los retos cambiantes del mundo laboral, y de aportar soluciones al desarrollo de su entorno y del país (Chacaltana y Sulmot, 2004). Conseguir esa aspiración sería un buen indicador de la calidad educativa en la IESTP, la Institución obtendría mayor prestigio y les daría seguimiento a sus egresados en las empresas y de esa manera poder contextualizarse y adaptarse a los nuevos cambios y ritmos de trabajo

En términos de Alcocer (2017) la lógica del mercado nacional formal está sujeto a documentos que acrediten mediante constancias, certificados, títulos u otros las habilidades desarrolladas en un determinado campo. En tanto para Bejarano y Gómez (2018), el seguimiento a los egresados y la eficiencia en los trámites disminuye el desempleo.

Existen puestos laborales para la inserción y empleabilidad, pero está condicionado a un documento que acredite los estudios que exige la normativizada de un país (Ibarra, 
2008). En tanto para López (2012) la empleabilidad de los egresados y recién graduados las condiciones pueden generar uno mismo y lograr ingresar a un puesto laboral. Sin embrago al momento de categorizar el honorario se ven limitados.

Las dificultades de obtener el título están inmersos a la dedicación laboral, que limita el tiempo para realizar una tesis (Pozo y Bretones, (2015). Para Torres (2019) la inserción laboral de los recién egresados es un reto de empezar una vida profesional con limitados habilidades que se obtiene en la práctica.

En la Figura 2 se muestra que al $61.5 \%$ alguna vez fue negada una oportunidad laboral por no tener título; en tanto, el $38.5 \%$ no tuvo impedimento alguno.

\section{Figura 2}

\section{Oportunidad laboral denegado}

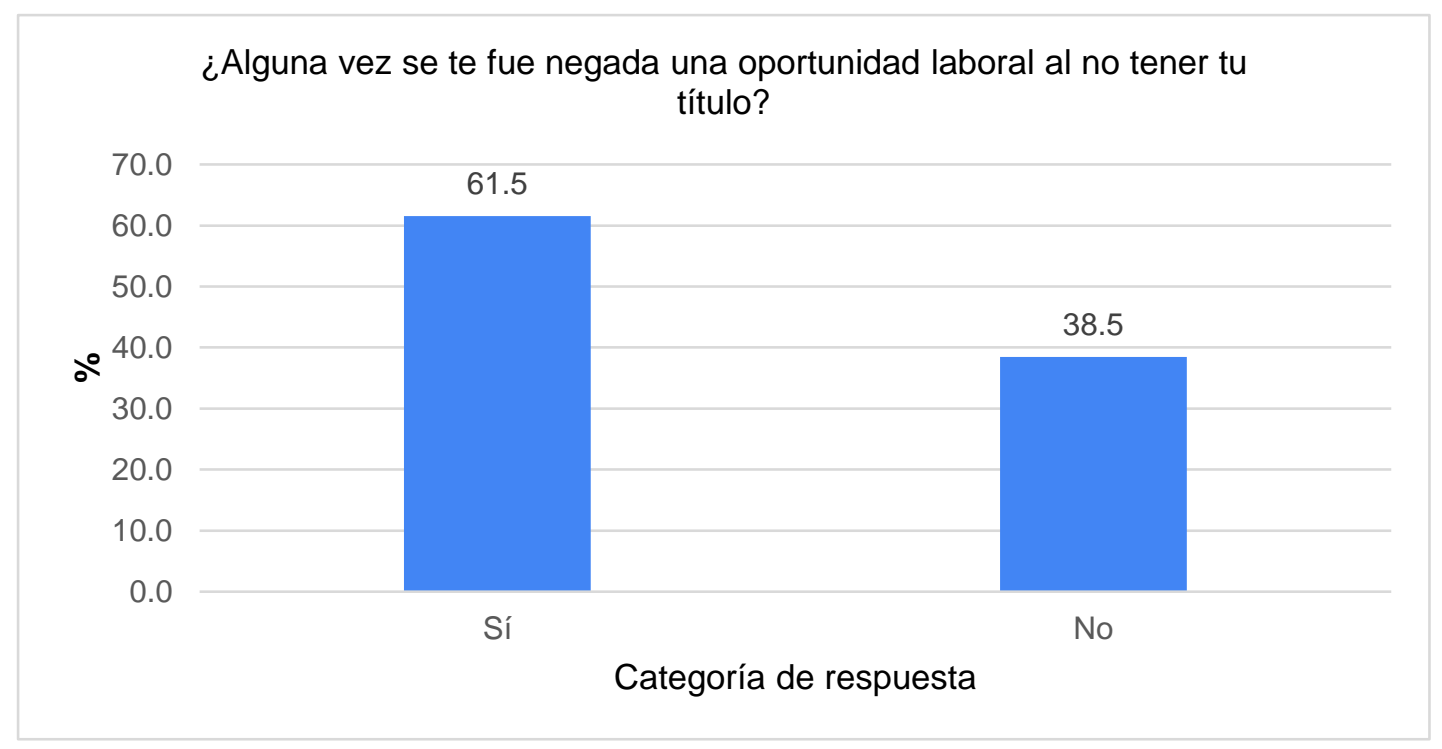

Es evidente la necesidad del título para insertarse laboralmente. Las organizaciones públicas y privadas suelen solicitar al postulante una evidencia que acredite los conocimientos requeridos para el puesto, así el título cobra un alto valor en la calificación que puede ser complementado con una prueba de conocimientos o entrevista personal (Sineace, 2015). La inexistencia del título genera la ssubcontratación y el desempleo (Carranza, 2019).

Las posibilidades laborales disminuyen para jóvenes egresados que por diversos motivos no lograron sacar en el debido momento un documento que acredite la culminación de sus estudios. (Aguirre, 2015). Este estudio refuerza Apaza (2017) que la educación y desempleo juvenil se vinculan al limitado acceso de estos documentos.

La calidad educativa no siempre está sujeto a la obtención de un documento como el título, más aún existen profesionales que sin poseer ninguna de estas son los más idóneos para puesto laborales (Espíritu y Ramírez, 2016). La acreditación y certificación en las instituciones de educación superior más aún generó exclusión que integración (Hernández, 2006).

En la Figura 3, se presenta las principales dificultades que tuvieron los entrevistados para adquirir el título. El $33.3 \%$ refiere que el elevado costo es el principal factor que 
dificulta el inicio del proceso; el $41.7 \%$ señala que la demora del trámite es el principal factor que retrasa el término del proceso de titulación; y el $25 \%$ indica no haber tenido dificultad alguna.

\section{Figura 3}

\section{Dificultades para obtener el titulo}

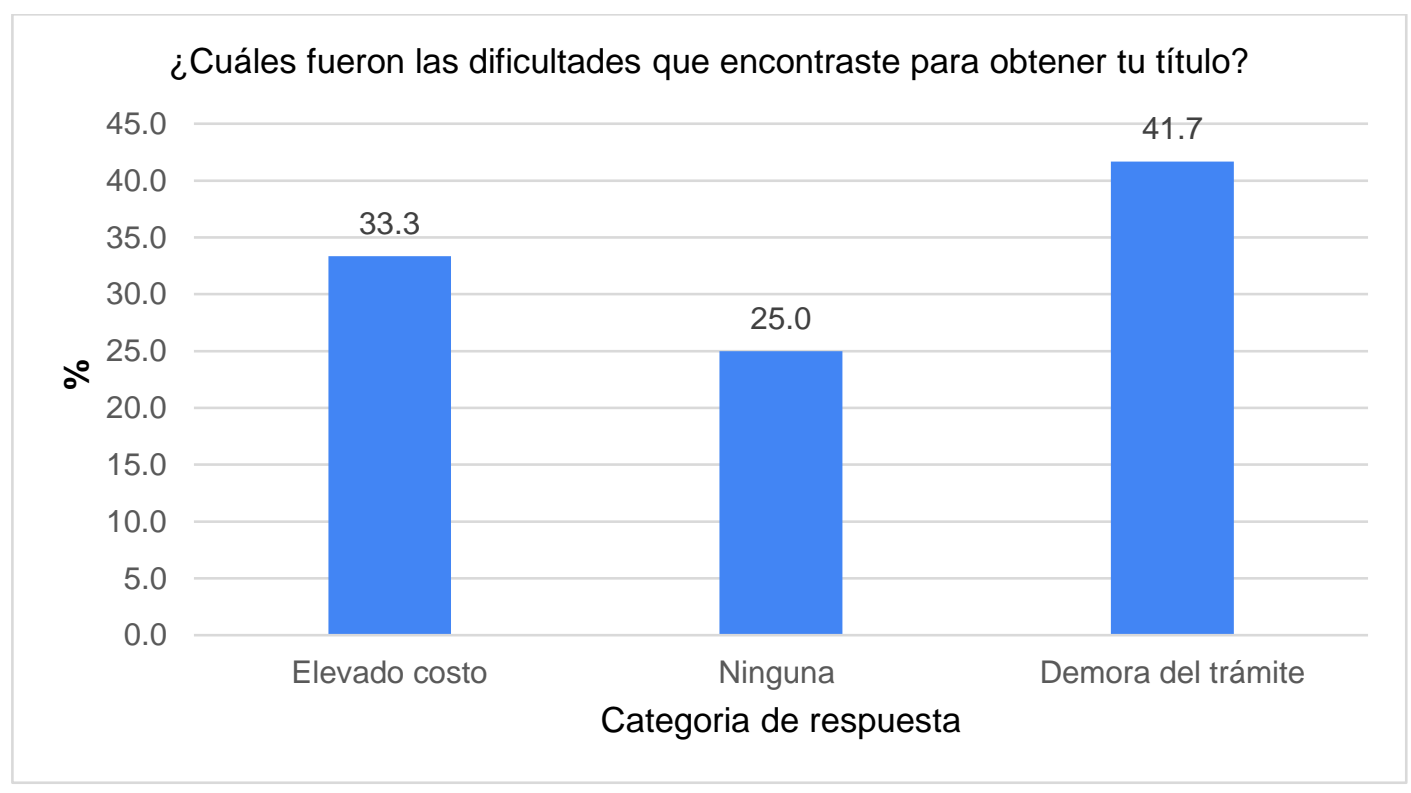

Los factores cruciales para el inicio y término del proceso de titulación están ligados a diversos aspectos: la atención de los administrativos; las exigencias establecidas por el Ministerio de Educación en relación con los requisitos; entre otros.

La Ley 29394 (31 de julio de 2009) no permitía que los egresados de los institutos tecnológicos obtengan oportunamente el título, dificultando su inserción laboral; por tal motivo, en el año 2015 se modificó con el reglamento de la Ley viabilizando la obtención oportuna del título (Decreto Supremo 010-2015-Minedu, 11 de agosto de 2015).

El Perú es el segundo país de la región donde los ciudadanos demoran en promedio 8.6 horas en completar un trámite. Esto se debe, entre otros factores, a que menos del $5 \%$ de los trámites se pueden realizar de forma digital; y a que un tercio de los trámites con el Estado no finaliza en una sola interacción, casi siempre hay que volver en reiteradas ocasiones (Banco Interamericano de Desarrollo, 2018).

Esta realidad nos lleva a reflexionar sobre la necesidad de evaluar el modelo de gestión de trámites en los institutos de educación superior tecnológica en relación con el tiempo que demora todo el proceso.

Existen experiencias exitosas para agilizar el proceso de trámite, tal es el caso de la Universidad Católica de Santa María que habilitó una plataforma on line para realizar los trámites con el fin de obtener el certificado del curso que realizan los estudiantes. Este proceso se puede implementar para las y los egresados. 


\section{CONCLUSIONES}

- El título es un requisito relevante para la inserción laboral tanto para empresas públicas y como privadas.

- El modelo de gestión condiciona la obtención del título dificultando la inserción laboral de egresados; sin embargo, se puede eximir de estos requisitos para la inserción laboral, esto conlleva aceptar el sueldo mínimo o por debajo de ello, no ascender a otros cargos y limitaciones en fortalecimiento de capacidades en otras instituciones.

- El elevado costo es el principal factor que dificulta el inicio del proceso de trámites para la titulación de los egresados.

- La demora del trámite es el principal factor que retrasa el término del proceso de titulación de los egresados.

\section{REFERENCIAS BIBLIOGRÁFICAS}

Aguirre, R. (2015). Análisis de la educación superior no universitaria en el Perú y su relación con el desarrollo económico nacional: la experiencia del Instituto Superior Tecnológico Privado Peruano de Sistemas "SISE" en Lima Metropolitana y los retos de la educación superior no universitaria. Tesis de pregrado. Lima, Perú.

Alcocer, P. (2017). Repercusiones del desempleo en jóvenes de Lima. Lima, Perú.

Apaza, E. (2017). Educación y desempleo juvenil en el distrito de Asillo, provincia de Azángaro, departamento de Puno en el año 2016. Puno, Perú.

Arévalo, R. (2016). Inserción en el campo laboral de los egresados de la carrera de Contabilidad del Instituto de Educación Superior Tecnológico Público de Amazonas de Yurimaguas. Tesis de pregrado. Perú.

Bejarano, C. y Gómez, M. (2018). Seguimiento a egresados de la escuela profesional de educación de la Universidad Católica de Santa María, Arequipa. Arequipa, Perú.

Carranza, E. (2019). Subcontratación y el desempleo en el Perú: análisis del periodo 2011 - 2017. Lima-Perú.

Chacaltana, R. y Sulmot, T. (2004) Retos cambiantes del mundo laboral. Perú.

Davies, R. y Dart, J. (2005). The Most Sgnificant Chang' [MSC] Technique.

Decreto Supremo 010-2015-Minedu (11 de agosto de 2015). Modifica el Reglamento de la Ley 29394, Ley de institutos y escuelas de educación superior.

Espíritu, F. y Ramírez, C. (2016). Calidad educativa con visión a la acreditación según el Sineace en una institución educativa de educación básica regular. Trujillo, Perú.

García, G. (2010). Empleabilidad, educación y equidad social. Argentina.

Hernández, A. (2006). La acreditación y certificación en las instituciones de educación superior hacia la conformación de circuitos académicos de calidad: ¿exclusión o integración? revista del centro de investigación. Universidad La Salle, vol. 7México. 
INEI (2017). Demografía en el Perú, la población en edad en trabajar. Lima, Perú.

Ibarra, S. (2008). Inserción laboral y empleabilidad en el mercado de trabajo en servicios sociales a la comunidad. Santiago de Chile.

Instituto de educación superior tecnológico privado red Avansys (2017). Reglamento institucional vra-reg-008-v01. Vicerrectorado Académico. Lima, Perú.

Instituto Nacional de Estadística e Informática de Perú (2007-2018). Población en edad de trabajar, según nivel de educación y ámbito geográfico.

Ley 28044 (28 de julio de 2003). Ley general de educación. Lima, Perú.

Ley 29394 (31 de julio de 2009). Ley de institutos y escuelas de educación superior. Lima, Perú.

López, M. (2012). La Empleabilidad y su incidencia en el nivel de empleo de los egresados y recién graduados de la Facultad de Ciencias Administrativas de la Universidad Técnica de Ambato. Tesis de pregrado. Ecuador.

Ministerio de Educación (2017). Ley de institutos y escuelas de educación superior y de la carrera pública de sus docentes. Decreto Supremo 30512

Ministerio de Trabajo y Promoción del Empleo - Produce (2012). Políticas nacionales de empleo. Lima, Perú.

Oficina de Coordinación Universitaria del Ministerio de Educación del Perú. (2004). Estructura y titulaciones de educación superior en Perú.

Pozo, C. y Bretones, B. (2015). Dificultades y retos en la implantación de los títulos de grado en las universidades españolas. España.

Ramírez, E. y Salas, G. (2011). Análisis de la Inserción Laboral de Estudiantes del IESTP Padah en el Periodo 2008/2010. Iquitos - Perú.

SINEACE, (2014). Calidad de la Educación desde la perspectiva del SINEACE. 2018, de Encuentro con Educadores. Lima, Perú.

Torres, U. (2019). La inserción laboral de los recién egresados universitarios en el Perú. LimaPerú. 